\title{
EL PAISAJE Y LA FORMA DE EXPANSIÓN EN LA CIUDAD DE LA PLATA Lineamientos y estrategias para la planificación del crecimiento urbano
}

\author{
Karina Jensen; Mariana Birche \\ Universidad Nacional de La Plata \\ Instituto de Investigaciones y Políticas del Ambiente Construido - CONICET \\ Director de proyecto de investigación: Leandro Varela \\ karinajensen_22@hotmail.com
}

\begin{abstract}
RESUMEN
La Plata es una ciudad planificada ex novo con un sistema de espacios verdes que materializaba las teorías higienistas del siglo XIX. Su trazado es racionalista, con diagonales y plazas cada seis cuadras logrando un equilibrio entre el espacio construido y el espacio público. Con los años la ciudad se expandió hacia terrenos productivos evidenciando una baja calidad urbana y paisajística que en adición a la falta de planificación y la carencia de espacios verdes genera un contraste con la situación dentro del casco urbano. La investigación pretende verificar que el crecimiento actual conformaría un entorno urbano fragmentado, tendiendo a la pérdida de la calidad paisajística. Se propone en una primera instancia identificar las características de la expansión urbana mediante un relevamiento exhaustivo del tejido que permita comprender las lógicas de expansión y las formas que configuran el paisaje. En una segunda instancia, se proponen lineamientos y estrategias de intervención.
\end{abstract}

Palabras clave: paisaje - expansión -planeamiento -ciudad

\begin{abstract}
La Plata is a city planned ex novo with a system of green spaces that materialized the hygienist theories of the nineteenth century. Its layout is rationalist, with diagonals and squares every six blocks achieving a balance between the built space and the public space. Over the years the city expanded into productive land evidencing a low urban and landscape quality that in addition to the lack of planning and the lack of green spaces generates a contrast with the situation within the planned urban area. The research aims to verify that the current growth would form a fragmented urban environment, tending to the loss of landscape quality. In the first instance, it is proposed to identify the characteristics of urban expansion by means of an exhaustive survey of the fabric that allows understanding the expansion logics and the shapes that make up the landscape. In a second instance, guidelines and intervention strategies are proposed.
\end{abstract}

Key words: landscape - sprawl - planning -city 


\section{Introducción}

A partir del año 2008, más de la mitad de la población mundial se encuentra viviendo en áreas urbanas y se estima que para el año 2030 el 60\% de la población vivirá en ciudades (datos según Banco Mundial). Además, cabe destacar que en América Latina, la urbanización supera en la mayoría de los casos el 90\% mientras que Argentina ya está superando el 94\%. En el país, Este acelerado proceso de producción de la ciudad se ha llevado a cabo con escasa regulación por parte del Estado, con insuficiente participación de las Universidades Nacionales y siguiendo principalmente las líneas impuestas por el mercado inmobiliario. Particularmente en la Región La Plata, se ha producido un gran crecimiento del cinturón agrícola en los últimos veinte años que se ve acompañado por la expansión de la mancha urbana, demostrando que la urbanización no solo se manifiesta a través de la cantidad de personas que viven en áreas urbanas sino también a partir de una relación que estas establecen con las áreas rurales.

El presente trabajo, se propone lograr una nueva perspectiva de la complejidad territorial en la región La Plata, a partir del proyecto de investigación "El paisaje como factor de mitigación de la degradación socio-ambiental en la Región La Plata" (acreditado 2014-2017 y 2017-2020 por la Secretaria de Ciencia y Técnica de la UNLP. Director: Mgr. Arq. Leandro Varela). El proyecto se desarrolla en la actualidad de manera interdisciplinaria y se posiciona como una nueva herramienta que se concibe desde la UNLP para los municipios de la región con miras a un desarrollo urbano más sustentable.

Es así como se plantea un abordaje de la región a partir del concepto central de paisaje, el cual permite trabajar en el territorio de manera multiescalar y transjuridisccional, estableciendo un carácter para cada unidad de paisaje y permitiendo la subdivisión de las unidades en los casos que sea necesario trabajar en otras escalas. Este proyecto ha servido para afianzar las relaciones entre investigadores de diferentes áreas disciplinares y plantear nuevos proyectos de investigación con bases comunes que permitan a su vez la integración entre municipios, ONGs, y diferentes universidades nacionales como entidades participantes de dichos proyectos.

Los problemas a los que se enfrentan los municipios, pueden derivar en situaciones de riesgo dentro de áreas urbanas. En este contexto, es necesario comprender que estas problemáticas han sido resultado de las políticas aplicadas, es decir que están construidas socialmente y por lo tanto se constituyen como escenarios que se desprenden del modelo de desarrollo. En este sentido, "En la medida que los daños causados al ambiente y a la sociedad por determinadas intervenciones antrópicas no son remediados tras su abandono, desmantelamiento, o cambio de actividad, puede suceder que sus efectos tiendan a permanecer en el tiempo e incluso a constituirse en factores de degradación aún más complejos o en condicionantes para nuevos desarrollos económicos o sociales". (GARAY, 2007)

Por lo tanto, el desarrollo territorial se puede concebir como un proceso de objetivos múltiples que, por su carácter y dinámica, demanda una adecuada planificación y gestión estratégica. A partir de esto, se entiende a la planificación como un "proceso de construcción de un proyecto colectivo capaz de implementar las transformaciones necesarias en la realidad que lleven a un futuro deseado". (BUARQUE, 1999)

A partir de aquí, las líneas de investigación se enfocan a guiar el proceso de planificación desde la perspectiva que brinda el concepto de paisaje, se busca la posibilidad de que al avanzar tecnológica y socialmente, el ambiente se recupere al mismo ritmo que la actividad humana lo afecte, a efectos de lograr una imagen superadora que integre las dimensiones estéticas, propias del proyecto paisajístico, y un resultado que vincule armónicamente la cuestión social, económica y ambiental de la región.

Para lograr esta vinculación, la investigación se trabaja transversalmente por medio del concepto integral de paisaje. Este concepto, es abordado por diferentes disciplinas, por lo que se pueden encontrar gran variedad de interpretaciones. También dentro de una misma disciplina es posible desarrollar el concepto de paisaje atendiendo en particular a un enfoque científico, social, cultural o estético. En el presente trabajo, se entiende al paisaje como:

El efecto de la superposición de la actividad humana sobre la naturaleza, e incluye las modificaciones derivadas de la actuación sobre el medio para hacerlo productivo - parcelaciones, áreas cultivadas, minería, forestación y deforestación, dehesas, pastos y ganaderías, etc.- y construir artificialmente sobre él, sean infraestructuras -puentes presas caminos, etc.- o realizaciones propiamente arquitectónicas. Se trata, por tanto, de un término que implica una orientación proyectual clara y una condición híbrida, natural y artificial: la proyección de la cultura- tanto en su significado etimológico como el convencional- sobre el territorio natural. (ABALOS, 2005)

Se puede afirmar entonces que, en este sentido, el concepto de paisaje posee un carácter proyectual claro y que, a su vez, los efectos estéticos que se derivan de él dependen de la visión de ciudad y del modelo de desarrollo. Es por eso que se orienta el trabajo a indagar sobre los aspectos subyacentes a los procesos de expansión urbana, que aparece principalmente configurada por numerosos proyectos particulares con escasa 
intervención estatal. En este contexto, resulta clave repensar las nuevas problemáticas urbanas desde una mirada que permita una nueva aproximación con miras de subsanar cuestiones sin resolver y orientar a la ciudad hacia un desarrollo urbano sostenible.

\section{El paisaje en la expansión urbana}

En Europa, la ciudad mediterránea se caracteriza por su compacidad y por la neta separación entre paisaje urbano y paisaje rural. Este fenómeno no depende solo de motivos de defensa sino, más en general del hecho que la sociedad urbana (la civitas) ha marcado durante milenios su distinción de la rural también en términos físicos, concentrándose en la urbs (Dematteis, 1996). Esta distinción entre paisaje rural y paisaje urbano, ha permitido desarrollar concepciones que difieren sustancialmente de la realidad latinoamericana, donde la separación entre lo rural y lo urbano se desdibuja debido a los procesos de dispersión urbana que tuvieron lugar en las últimas décadas. Al respecto, resulta interesante señalar los numerosos aportes metodológicos (Observatorio del paisaje de Cataluña, l'Agence française pour la biodiversité, The Countryside Agency, entre otros) producidos por países europeos con mayor tradición paisajística donde se pone el foco en cuestiones como las cuencas visuales, que responden a la topografía local y también a la escala territorial de las unidades de paisaje con las que se trabaja.

Las periferias de las ciudades latinoamericanas están compuestas por espacios social y culturalmente heterogéneos, donde conviven tanto los sectores más pobres de la población como los de mayor nivel adquisitivo. A este fenómeno se le suma el desplazamiento de sectores de la población con alto nivel socio económico a urbanizaciones cerradas, las cuales funcionan como guetos urbanos aislados del tejido. Cada vez más ciudadanos buscan una organización privada y eficiente de su vecindario que les provea de los servicios que antes solían ser públicos. En la década del '90, Pérgolis (1998) señalaba para las grandes ciudades latinoamericanas, que "la ciudad total está siendo sustituida por la ciudad fragmentada"; es decir, el proceso de segregación socioespacial, llevado a extremas consecuencias. En este contexto, en Latinoamérica "la nueva ciudad de la dispersión está surgiendo de forma acrítica, más preocupada por las urgencias de lo inmediato que por comprender su propio sentido" (Arias Sierra, 2003).

Estos procesos de expansión conllevan también cambios en el paisaje. En este sentido, Joan Nogué (2007) señala que "nunca habíamos transformado el territorio a la velocidad en la que lo hemos hecho en los últimos decenios". Si bien esta afirmación la hace en relación al contexto español, se puede generalizar para describir las transformaciones a nivel global y en particular las ciudades de Latinoamérica, donde se han experimentado transformaciones que se dan en el marco del proceso de restructuración del sistema capitalista del último cuarto de siglo XX.

A partir de finales del siglo XX en Argentina, el Estado modifica sus acciones en pos de posibilitar y facilitar grandes inversiones en la ciudad, de capitales nacionales y extranjeros. Para ello se iniciaron procesos de desregulación político-administrativa del suelo urbano, que incrementaron la participación del mercado en la configuración de las ciudades (Clichevsky, 2001).

Las ciudades deben resistir en el largo plazo los efectos de la lógica cortoplacista, que por un lado suman el incremento de población, de espacio construido, de automóviles, de actividades y de estrés urbano. Y por otro, resta cantidad de espacios verdes públicos, de espacios colectivos, de espacios para los peatones, de asoleamiento, de superficies de suelo absorbente, de forestación. La aplicación de políticas urbanas orientadas a partir de la priorización de la lógica económica arroja como resultante la pérdida de paisaje y de calidad de vida urbana. Los cambios en la dinámica del paisaje ya sea inducido por la acción humana o por parte de procesos naturales alteran la estructura y función de este.

Intervenir el territorio desde una mirada paisajística implica ser consciente de las transformaciones que en él se han dado y la importancia de pensar en las generaciones futuras. Implica también, reconocer la estrecha relación entre cultura y naturaleza, donde lo más importante es construir nuevos vínculos entre el hombre y el medio que lo rodea, en pos de establecer una relación armónica con el ambiente natural y reducir la vulnerabilidad frente a los fenómenos críticos que afectan la región. Por lo tanto, pensar nuevas estrategias desde la mirada del paisaje es pensar en una manera integral de ver y actuar en el territorio, teniendo en cuenta no sólo la dimensión física ambiental, sino también la dimensión social, cultural y estética con el fin de mejorar la calidad de vida de la población.

Es así como se entiende al concepto de paisaje continuo como una oportunidad de integrar la ciudad. Hablar de una visión integral que permita ver más allá, es mirar las interrelaciones que se dan entre los sistemas y entender el problema desde un punto de vista reflexivo. Esto plantea una nueva forma de aproximación la ciudad que permite actuar sobre ella pero entendiendo que está llamado a formar parte de un ecosistema en 
constante transformación. El desafío más importante consiste en planificar coordinadamente tiempos diferenciados como lo son el tiempo humano y el tiempo natural, pensando siempre en la idea de totalidad De esta forma, el paisaje se constituye como el protagonista de las profundas transformaciones sociales y morfológicas que configuran el proceso de expansión.

\subsection{La región de estudio}

El área de estudio se encuentra inserta en la región del Gran La Plata la cual constituye un conglomerado urbano alrededor de la ciudad de La Plata, capital de la provincia de Buenos Aires, Argentina. El Gran La Plata está compuesto por los partidos de La Plata, Ensenada y Berisso y es la sexta aglomeración más poblada del país. Se encuentra al nordeste de la Provincia de Buenos Aires, y muy cerca del límite sudeste del Gran Buenos Aires.

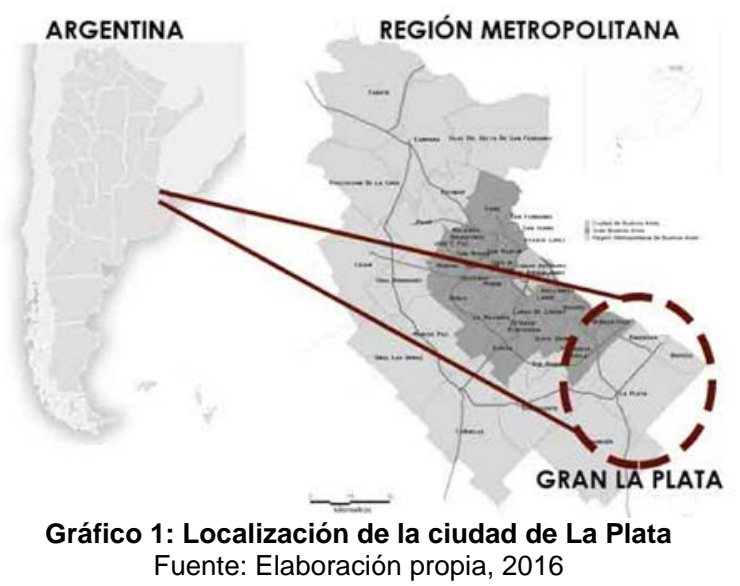

La expansión del área urbana en la región La Plata se produjo de manera vertiginosa principalmente en los últimos veinticinco años. Este fenómeno, impulsado (entre otras cosas) por los mecanismos del mercado, sumado a la imposibilidad de acceso al suelo urbano, produce una expulsión de la población hacia terrenos más alejados del centro (suelo productivo), lo que favorece la expansión de las periferias y la consolidación de los barrios más alejados. Este crecimiento hacia los terrenos productivos de la ciudad, configurando espacios urbanos fragmentados, que evidencian una baja calidad urbana y paisajística en estas nuevas zonas periféricas.Esta situación, en adición a la falta de planificación y la carencia de espacios verdes públicos genera un fuerte contraste con la situación dentro del casco urbano (Jensen y Birche, 2017).

En la década de los ' 90 , tuvieron lugar distintos procesos que dinamizaron la configuración urbana de ciudad de La Plata, particularmente el eje noroeste. Entre estos procesos, la inauguración de la Autopista La Plata - Buenos Aires (1996) y la readecuación de la Autovía 2 (1993-99), han favorecido la aparición de nuevos emprendimientos como barrios cerrados que propician la fragmentación socio-espacial de la ciudad. Estos lugares, funcionan como ciudades en miniatura y poseen todo un sistema de "espacio público" en su interior, independiente del sistema de espacios públicos de la ciudad. Conjuntamente, el surgimiento de los hipermercados y la configuración de grandes superficies comerciales aisladas del entorno urbano también han contribuido a conformar nuevos ámbitos de uso público bajo el dominio de la propiedad privada. Si bien se han realizado mejoras y remodelaciones de los centros comerciales tradicionales y se han sancionado normativas al respecto, como el Código de Espacio Público en 2004, estas acciones no terminan de conformar una base sólida sobre la cual el espacio público se consolide como estructurador de la ciudad, especialmente en las zonas periféricas.

\subsection{La cuestión urbana en la región La Plata}

El área de estudio se encuentra en la región pampeana la cual es uno de los territorios agroproductivos más importante del mundo. La región metropolitana de buenos aires se ha expandido vertiginosamente en los últimos 25 años sobre territorios productivos, constituyendo a la región pampeana como uno de los territorios más antropizados de la Argentina.

La región de la Plata también ha experimentado un crecimiento exponencial desde la década del 90, impulsado (entre otras cosas) por los mecanismos del mercado y la imposibilidad de acceso al suelo urbano. Este fenómeno produce una expulsión de la población hacia terrenos más alejados del centro (suelo productivo), lo que favorece la expansión de las periferias y la consolidación de los barrios más alejados. 
Estos procesos de urbanización no constituyen en la actualidad una forma conveniente para conformar un entorno urbano que garantice la calidad de vida de la población, ni tampoco un modelo adecuado de crecimiento para la ciudad ya que ha adoptado la morfología de una ciudad difusa.

En contraposición, en un modelo de crecimiento que responda al concepto de ciudad compacta, la mayor integración tanto física como socio-espacial, se traduce en una mejor convivencia entre los diferentes grupos sociales, pudiéndose abordar mejor los problemas de integración, e incluso compartir mejor el diagnóstico y las soluciones en materia de inseguridad ciudadana. Asimismo, el modelo de urbanización compacta, al corresponderse con el de una ciudad caracterizada por la mezcla de actividades y servicios, facilita los desplazamientos a pie y el intercambio y relación entre sus habitantes, debido a la mayor proximidad resultante de la concentración en el espacio unidades de características diferentes. La estrategia de la ciudad compacta de aumentar la complejidad, sin necesidad de aumentar sustancialmente el consumo de materiales, suelo y energía, es la alternativa más sostenible en cuanto a organización y eficiencia del consumo energético. En este contexto, el crecimiento de las periferias sin ningún tipo de regulación conlleva a que sectores de la población vivan en zonas donde los servicios o infraestructuras son inadecuadas. De esta forma, se constituye la ciudad como un homogéneo monofuncional donde el uso predominante es el residencial. Estas nuevas zonas de la periferia no están dotadas de espacios verdes, servicios, infraestructura, espacios para la recreación, en otras palabras no se consolidan como una subcentralidad.

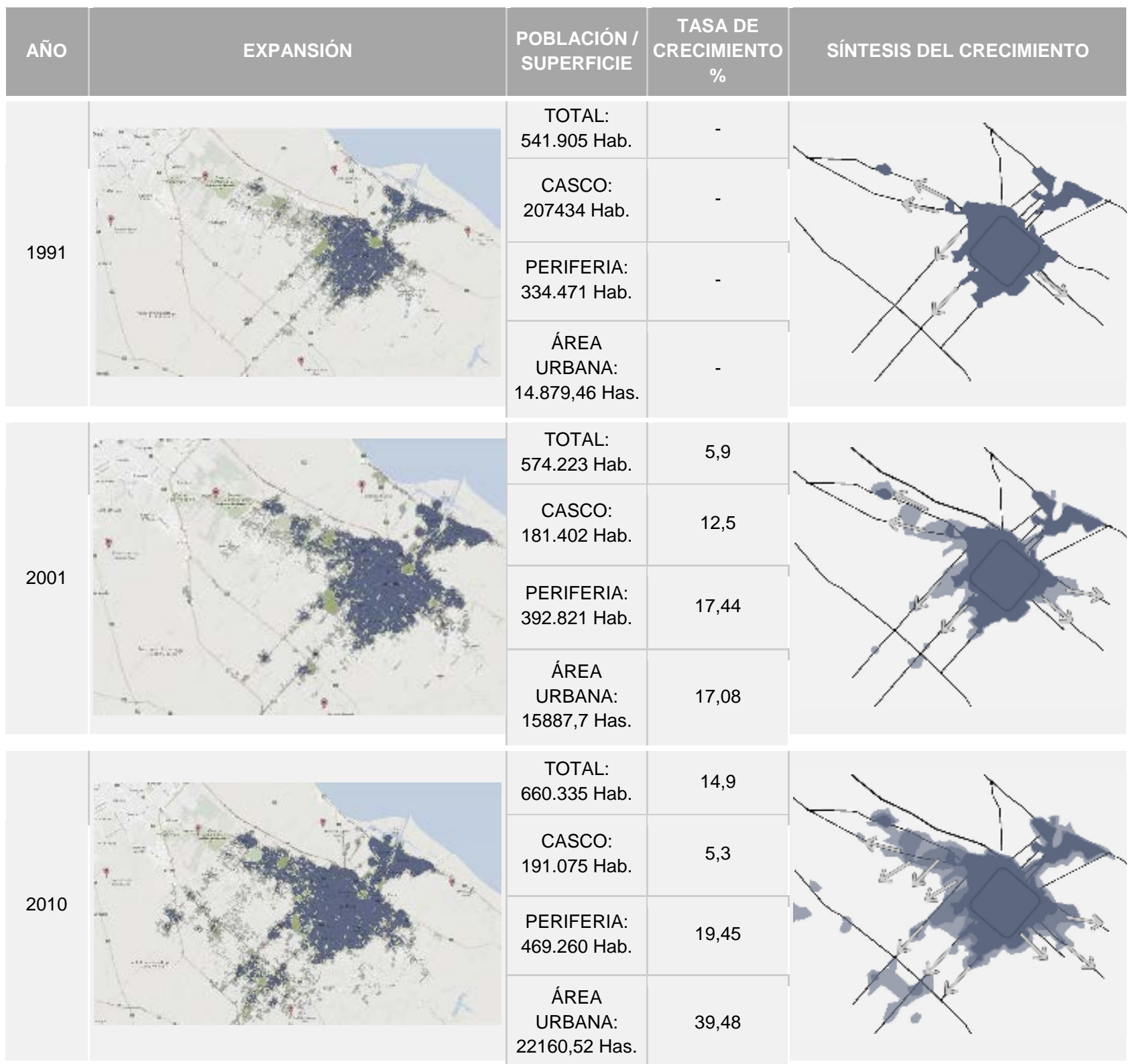


Gráfico 2: Expansión del área urbana construida en los últimos 25 años.

Fuente: Elaboración propia en base a los censos 1991, 2001, 2010 y al atlas de crecimiento urbano CIPUV, 2016

Como se mencionó anteriormente la expansión urbana en la Plata en los últimos 30 años ha sido exponencial (Gráfico 2), según el Censo 1991, la ciudad de La Plata tenía 541.905 habitantes donde 334.471 (más de la mitad) reside en la periferia. Esta diferencia del casco y la periferia se incrementó, para el 2010 la gente que vive en la periferia es de $36,98 \%$ y se ha sostenido a lo largo de las décadas, mientras que en el casco la población decreció un 12,5\% en 2001 y luego solo creció un 5,3\% para 2010. El crecimiento se ha dado principalmente sobre el eje noroeste y también en sentido suroeste.

\section{Abordaje metodológico}

Para abordar la presente investigación, la metodología adoptada identifica los distintos tipos de tejido en relación a la estructura urbana existente y a la disposición de los principales usos del suelo. Es por ello que para la identificación de los tipos de tejido se ha elaborado una metodología basada en la fotointerpretación de imágenes satelitales de Google Earth (año 2017). Se tienen en cuenta como parámetros la densidad, porcentaje de ocupación de la manzana, la cobertura de infraestructuras y servicios del área. Para realizar el relevamiento se elabora una matriz de datos, mientras que para la sistematización y análisis de la información se utiliza un Sistema de Información Geográfica (SIG).

\begin{tabular}{|c|c|c|c|}
\hline DIMENSIONES & $\begin{array}{l}\text { TIPOS DE } \\
\text { TEJIDO }\end{array}$ & INDICADORES & EJEMPLO \\
\hline \multirow{5}{*}{$\begin{array}{l}\text { CIUDAD } \\
\text { FORMAL }\end{array}$} & $\begin{array}{l}\text { Áreas centrales } \\
\text { y } \quad \text { Barrios } \\
\text { consolidados }\end{array}$ & $\begin{array}{l}\text { Densidad residencial entre } 100 \text { y } 300 \\
\text { habitantes/hectárea. Trama urbana regular, con } \\
\text { cobertura de infraestructuras urbanas, } \\
\text { equipamientos y espacios públicos. Estructura } \\
\text { parcelaria rígida. Intensa ocupación de las } \\
\text { parcelas. En las áreas centrales, proceso de } \\
\text { verticalización edilicia, terciarización y pérdida de } \\
\text { población residente. }\end{array}$ & 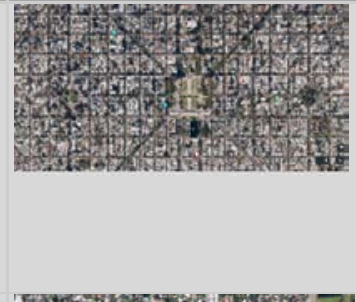 \\
\hline & $\begin{array}{l}\text { Loteo formal con } \\
\text { diferentes } \\
\text { grados de } \\
\text { ocupación }\end{array}$ & $\begin{array}{l}\text { Densidad neta aproximada: } 60 \text { a } 130 \\
\text { habitantes/hectárea. Trama urbana regular, con } \\
\text { déficit de cobertura de infraestructuras urbanas, } \\
\text { equipamientos y espacios públicos. Parcelas con } \\
\text { menor intensidad de ocupación y parcelas baldías. }\end{array}$ & \\
\hline & $\begin{array}{l}\text { Grandes } \\
\text { parcelas } \\
\text { residenciales }\end{array}$ & $\begin{array}{l}\text { Densidad neta aproximada: menos de } 40 \\
\text { habitantes/hectárea. Sectores de segunda } \\
\text { residencia o residencia principal, sobre grandes } \\
\text { parcelas con acceso directo desde la trama vial. En } \\
\text { general, sin provisión de servicios públicos de } \\
\text { agua y cloacas }\end{array}$ & \\
\hline & $\begin{array}{l}\text { Urbanizaciones } \\
\text { residenciales } \\
\text { cerradas }\end{array}$ & $\begin{array}{l}\text { Densidad neta aproximada: menos de } 40 \\
\text { habitantes/hectárea.Loteos privados de grandes } \\
\text { parcelas, con un acceso restringido y calles } \\
\text { internas de estructura arborescente. Según las } \\
\text { reglamentaciones urbanas locales, pueden llegar a } \\
\text { contar con servicios sanitarios administrados por el } \\
\text { consorcio o la sociedad desarrolladora. }\end{array}$ & \\
\hline & $\begin{array}{l}\text { Vivienda social } \\
\text { media densidad }\end{array}$ & $\begin{array}{l}\text { Densidad neta aproximada: entre } 300 \text { y } 600 \\
\text { habitantes/hectárea. Conjuntos habitacionales de } \\
\text { vivienda pública sobre grandes parcelas indivisas, } \\
\text { edificios de } 2 \text { a } 4 \text { pisos, sin ascensor, entre } 500 \text { y } \\
1.000 \text { viviendas. Trazado generalmente } \\
\text { discontinuo respecto de la trama, con provisión de } \\
\text { servicios sanitarios pero con dificultades de } \\
\text { administración y mantenimiento. }\end{array}$ & 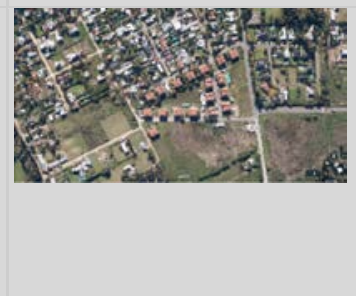 \\
\hline
\end{tabular}




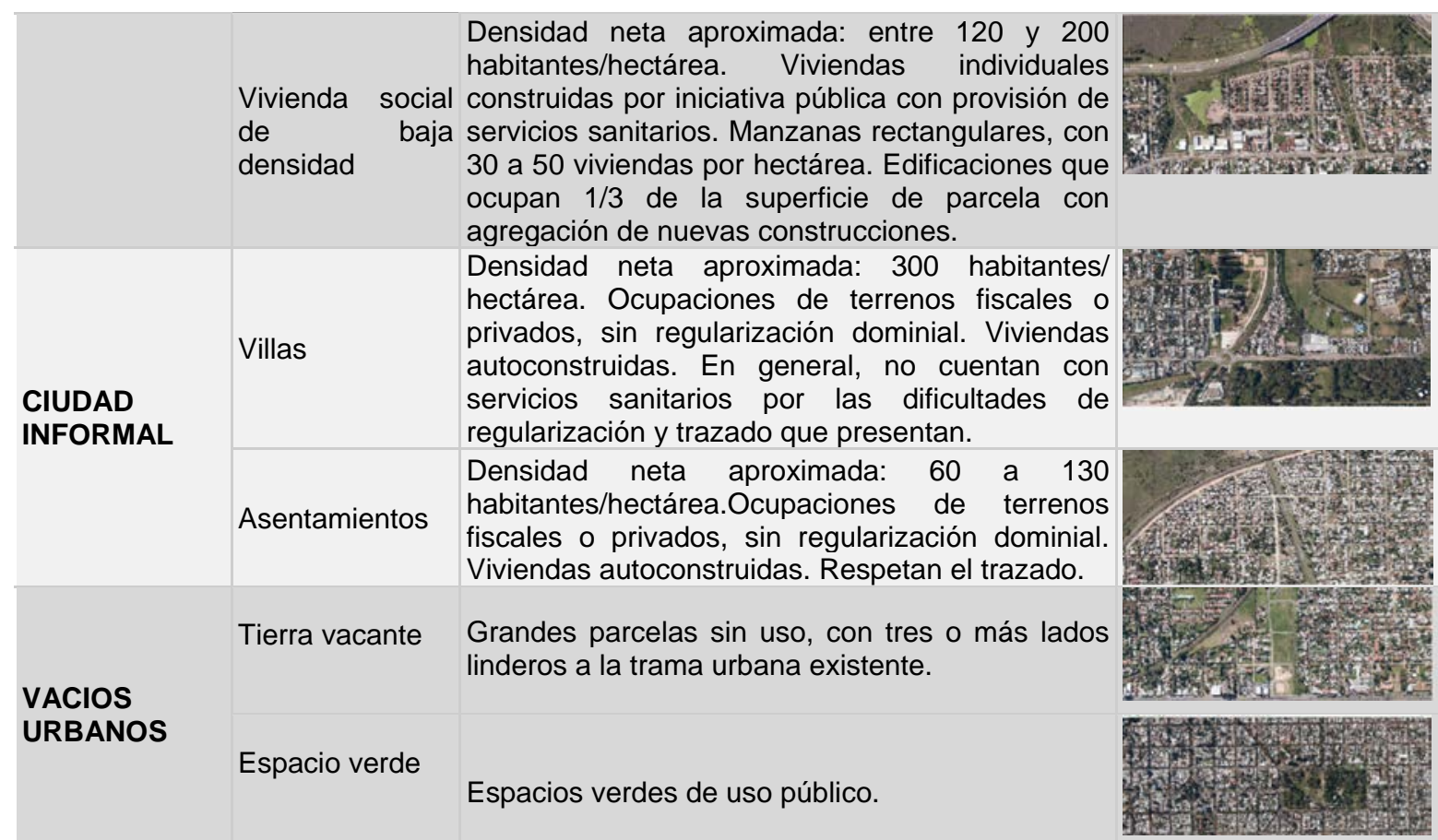

Gráfico 3: Tipos de tejido. Fuente: elaboración propia, 2017.

A partir de comprender e identificar los distintos tipos de tejidos, se observa que la ciudad está compuesta por estas "piezas" donde se evidencia la fragmentación que existe en el área urbana. Se elabora un mapa en donde se observan los tres tipos de consolidación del área residencial, las urbanizaciones cerradas, villas y asentamientos y los grandes vacíos urbanos.

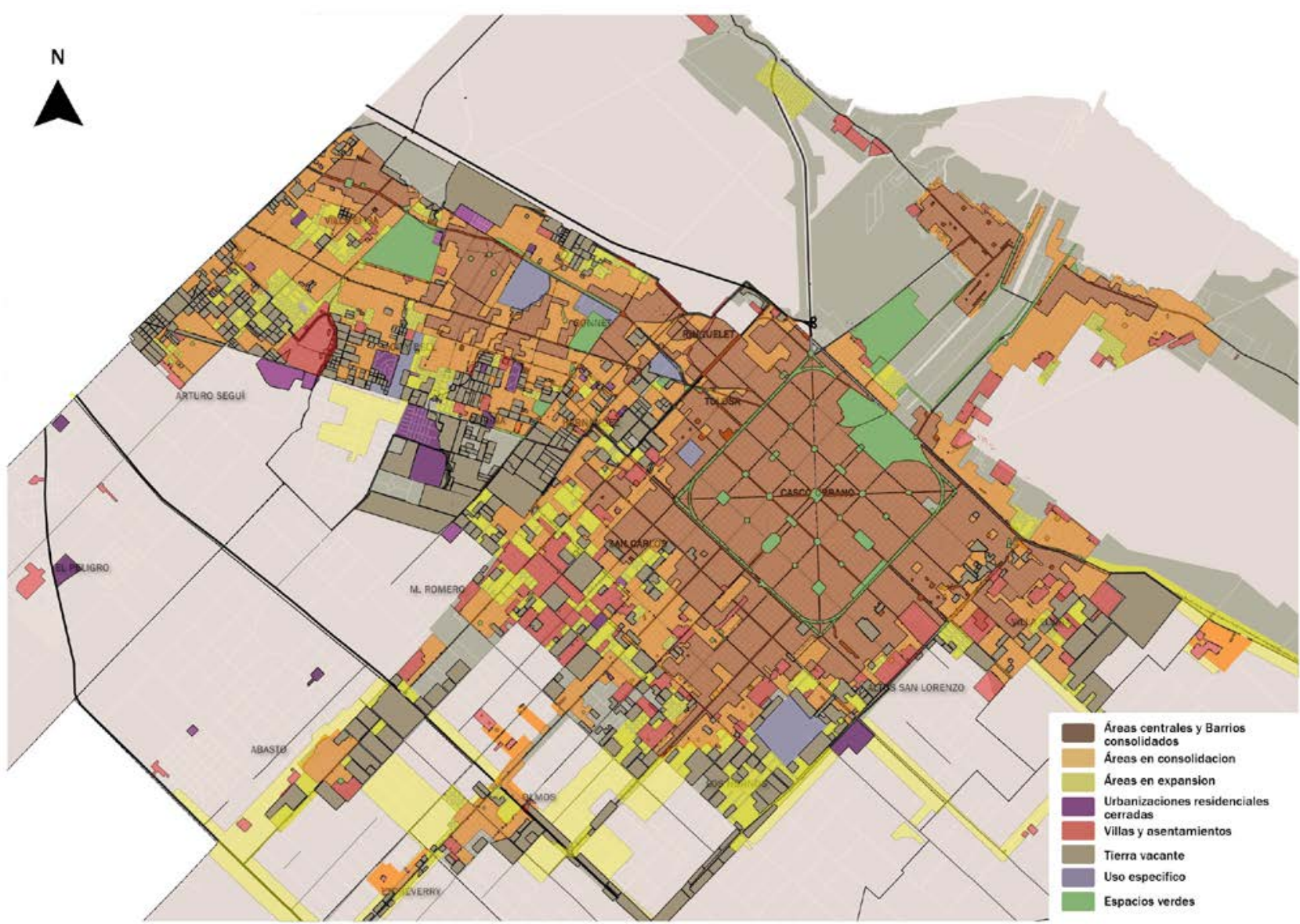

Gráfico 4: Tipos de tejidos. Fuente: elaboración propia, 2017. 
Se puede decir entonces que no se observa un crecimiento equilibrado ni tampoco compacto y que el área urbana se encuentra fragmentada, exceptuando el casco fundacional que presenta una situación más homogénea y consolidada como así también el primer anillo de desborde de este. Al observar la periferia se evidencia la fragmentación existente y se identifican zonas donde predominan las villas y asentamientos como lo es el sector sur este y zonas donde predominan las urbanizaciones cerradas como lo es el eje noroeste. En cuanto a los vacíos urbanos se observa la falta de espacios verdes que hay la periferia y la presencia de tierras vacantes.

Luego de analizar los tipos de tejido, se procedió a identificar las unidades de paisaje en la región. Se denomina unidad de paisaje al área geográfica con una configuración estructural, funcional o perceptivamente diferenciada, única y singular, que ha ido adquiriendo los caracteres que la definen tras un largo período de tiempo. Estas unidades son áreas del territorio que presentan un carácter paisajístico diferenciado. La evolución que sufre el territorio por los factores naturales y antrópicos que le dan un rasgo distintivo cada una. "Las unidades de paisaje son porciones del territorio con un mismo carácter, es decir, están caracterizadas por un conjunto de elementos que contribuyen a que un paisaje sea diferente de otro, y no por ello mejor o peor".

La definición de las unidades se basa en elementos paisajísticos perdurables en el tiempo.

Las seis variables paisajísticas que sirven para delimitar las unidades de paisaje son:

- Los factores fisiográficos y, en especial, el relieve.

- Los usos y las cubiertas del suelo, cuyo estudio permite identificar la disposición espacial característica de cada paisaje.

- La dimensión histórica del paisaje, es decir el factor humano como principal agente transformador del paisaje desde una perspectiva histórica. El paisaje es un reflejo de las funciones que secularmente se le han ido otorgando. Se fundamenta en las formas y estructuras paisajísticas que hemos heredado, como por ejemplo los patrones urbanos, la estructura parcelaria y de la propiedad, las tipologías de asentamiento en el territorio, la distribución histórica de determinadas actividades productivas, las infraestructuras hidráulicas, o la red de caminos antiguos u otras infraestructuras de comunicación y transporte. La pervivencia de estas huellas en el territorio contribuye a definir el carácter de un determinado paisaje y la identidad de un territorio y son elementos básicos que deberán tenerse en cuenta en la ordenación posterior del paisaje.

- La estructura del paisaje debería ser analizada desde la ecología del paisaje

- El estudio de visibilidades y, por lo tanto, la visión del paisaje. Este aspecto es muy importante para la definición de las unidades de paisaje, ya que la observación y comprensión del paisaje está fuertemente condicionada por la existencia de unos ciertos puntos de observación y de recorridos visuales que son los que en mayor medida contribuyen a la percepción del paisaje.

- Detección de dinámicas recientes y tendencias inmediatas. La definición de las unidades se debe basar también en elementos paisajísticos perdurables en el tiempo.

- La identidad del lugar. El paisaje es mucho más que un conjunto de componentes naturales, estéticos o el resultado de unos factores históricos y socioeconómicos que han intervenido sobre el territorio. El paisaje es, en gran medida, un elemento vivencial, es decir, que está configurado a partir de la suma de experiencias vividas a lo largo de los años y en el que arraigan sentimientos de pertenencia e identidad. La relación social y económica ancestral establecida entre distintas poblaciones de un territorio, o entre un pueblo y un río cercano, el uso de ciertas técnicas y prácticas de manejo de los cultivos, la denominación de los lugares, la existencia de un dialecto hablado en un pequeño territorio o el reconocimiento especial de ciertos paisajes son, entre otros, algunos factores que nos identifican con un determinado paisaje.

En el área de estudio, se identifican las áreas que poseen un mismo carácter paisajístico:

1 | Costa: es el área comprendida por el Río de la Plata hasta la orilla del mismo.

2 | Fluvial: comprende todos los canales y arroyos de la región, su respectivo cauce y planicie de inundación. 3 | Humedal: área comprendida entre el albardón costero y la terraza alta. Se caracteriza por ser zona de bañados.

4 | Urbano: área que comprende la zona urbana. En la misma se reconocen la ciudad de la Plata, Berisso y Ensenada.

5 | Periurbano: área que comprende la zona que queda entre lo urbano y el frutihorticola.

6 | Frutihorticola: Área delimitada por fuera de la expansión urbana, que forma parte del cordón frutihortícola y se corresponde con el uso de suelo rural.

7 | Industrial: Comprende a la zona donde se localizan la mayoría de las industrias en la región, en el puerto y sobre la ruta 36 . 


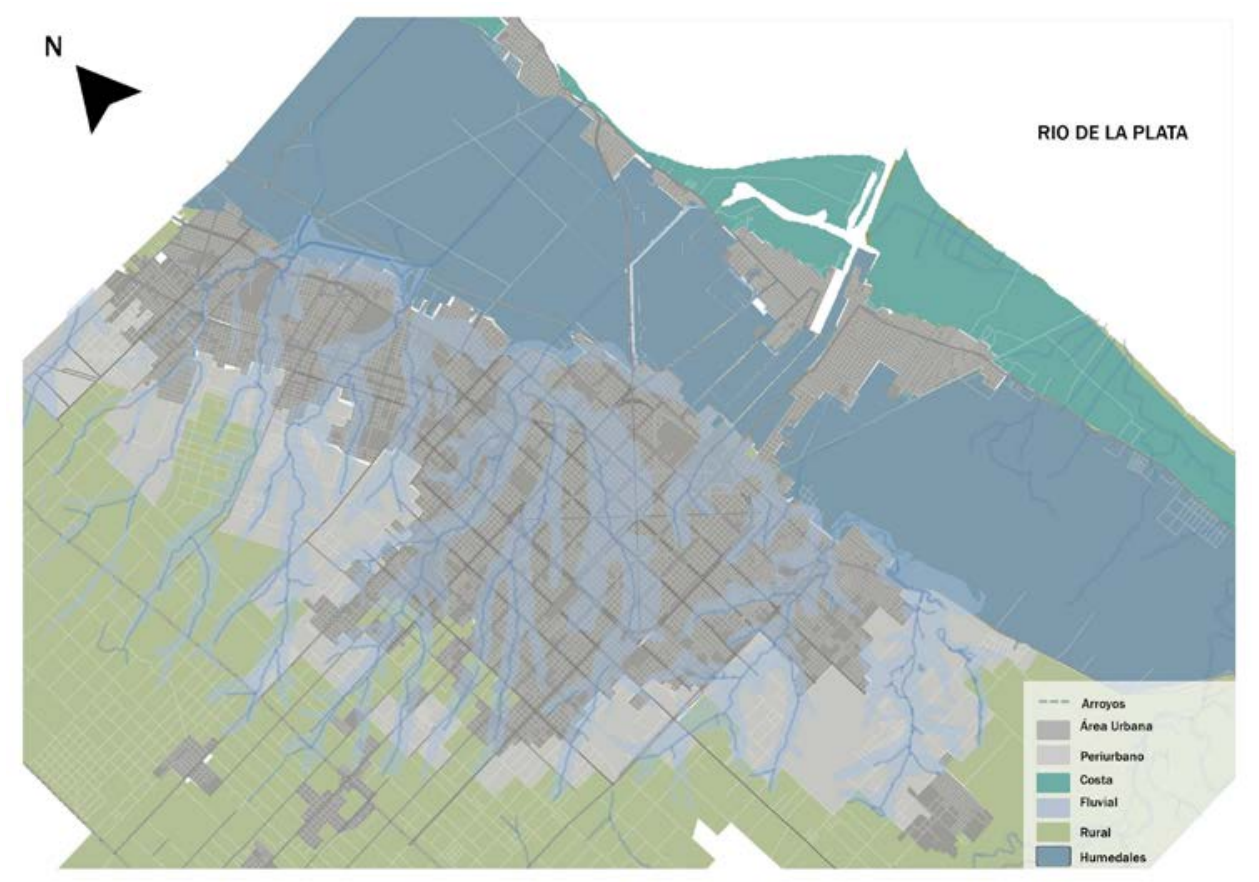

Gráfico 5: Unidades de Paisaje en la región del Gran La Plata. Fuente: elaboración propia, 2017.

En base a los tipos de tejido identificados en correlación con las unidades de paisaje, se proponen lineamientos para el ordenamiento y la gestión del crecimiento urbano de la ciudad los cuales tenderían a reducir la fragmentación existente. Estos se basan en la puesta en valor de los atributos paisajísticos y el fortalecimiento de cada pieza urbana según su función específica. De esta forma, se proponen los siguientes lineamientos:

-Fortalecer las nuevas subcentralidades

-Fomentar el completamiento de la trama urbana en zonas consolidadas y en consolidación

-Reestructuración y jerarquización de vías de circulación

-Adecuar los espacios públicos existentes con miras a fortalecer las características propias del paisaje urbano platense.

-Fomentar la refuncionalización de tierras vacantes como espacios verdes integrados al sistema de espacios públicos.

-Delimitar el sector periurbano para la localización de viviendas y los indicadores para este.

-Relocalizar y/o adecuar viviendas en zonas inundables según su localización en planicies de inundación hidrológica o topológica.

-Delimitación de un cordón productivo regulado por indicadores y el Coeficiente de Absorción del Suelo (CAS). -Convertir las planicies de inundación de los arroyos en el área urbana en espacios verdes integrados al sistema de espacios públicos.

\section{Algunas reflexiones finales}

Como se ha mencionado anteriormente, las zonas periféricas de la ciudad han desempeñado un rol protagónico en lo que respecta al crecimiento urbano, recibiendo aproximadamente el $70 \%$ del crecimiento demográfico desde 2010 a la fecha. Estos procesos de urbanización se hacen visibles no solo a través del aumento poblacional sino que modifican sustancialmente la relación que mantiene la ciudad con el campo y los elementos naturales, particularmente los cursos de agua. A través de los años, se han configurado grandes zonas periurbanas que adquieren los rasgos particulares de una zona de transición y a la vez sirven de apoyo a la ciudad, pero también son susceptibles de ser absorbidas por el crecimiento urbano. La periferia platense se caracteriza por su dinamismo y su inmersión en los procesos de cambio morfológicos, funcionales y sociales. Esto constituye un valor distintivo del área periférica y la posiciona estratégicamente como un área de oportunidad.

Sin embargo, se puede afirmar que el ritmo de los procesos de ocupación resulta ampliamente superior al de cualquier tipo de planificación por parte del estado. Esta situación ha derivado en una gran cantidad de espacios fragmentados, desprovistos de ciudad y de paisaje. En este sentido, creemos que mantener una 
visión integral de la problemática y trabajar con la amplitud conceptual necesaria resulta clave al momento de estudiar la ciudad. Es por eso que sostenemos que las nuevas formas de apropiación social y la adaptación de los espacios de esparcimiento y recreación obligan a los investigadores a reformular presupuestos y a reorientar las líneas de trabajo con una mayor conciencia respecto de la realidad urbana y generando estrategias de proyecto basadas en la compresión de las lógicas de intervención actuales.

Para finalizar, desde nuestro Instituto creemos que se dispone de una experiencia acumulada en las instituciones locales en relación a los procesos de generación, acumulación e intercambio de conocimiento y que lentamente se van incorporando nuevas metodologías de análisis de los procesos urbanos, incluyendo enfoques de participación y de desarrollo de capacidades a partir de alianzas entre actores con una visión emprendedora y transformadora de la sociedad. Sostenemos que la riqueza y la finalidad de los proyectos de investigación se centran no solo en la producción misma de conocimiento sino en poder integrar el saber académico con la realidad, asistiendo a las prácticas de gobierno locales con conocimiento local a fin de concretar acciones tendientes a un desarrollo urbano sostenible.

\section{Bibliografía}

-ARIAS SIERRA, P. (2003). Periferias y Nueva ciudad. El problema del paisaje en los procesos de dispersión urbana. Sevilla: Secretariado de publicaciones de la Universidad de Sevilla

-BAHRDT, H. (1970); La moderna metrópoli: reflexiones sociológicas sobre la construcción en las ciudades. Buenos Aires: Eudeba

-Convenio Europeo del Paisaje (2000). Florencia.

-DEMATTEIS, G. (1996). Suburbanización y periurbanización Ciudades anglosajonas y ciudades latinas La ciudad dispersa. Urbanitats, 4.

-JENSEN, K. y BIRCHE, M. (2017) Espacio verde: Elemento clave en la gestión urbana. Revista Ciudades (México), 0187-8611.

-RAVELLA, O. (2001). La planificación urbana regional. La Plata: UNLP

-RAVELLA, O. y VARELA, L. (Comp.) (2008). Diseñando el paisaje. Bs.As: Prometeo.

-SERVICIO DE EVALUACIÓN DE IMPACTO AMBIENTAL (2013) Guía elaborada por el Departamento de Estudios y Desarrollo de la División de Evaluación Ambiental y Participación Ciudadana del Servicio de Evaluación Ambiental de Chile. 